Supporting Information

\title{
Non-enzymatic Glucose Sensing and Magnetic Property Based on the Composite Formed by Encapsulating Ag Nanoparticles in Cluster-Based Co-MOF
}

Yang Liu ${ }^{\dagger \neq}$, Wen-Juan Shi ${ }^{\dagger}, \mathrm{Yu}-\mathrm{Ke} \mathrm{Lu}^{\dagger}$, Ge Liu ${ }^{\dagger}$, Lei Hou ${ }^{*}, \dagger$ and Yao-Yu Wang ${ }^{\dagger}$

${ }_{\dagger}$ Key Laboratory of Synthetic and Natural Functional Molecule Chemistry of the Ministry of Education, National Demonstration Center for Experimental Chemistry

Education (Northwest University), Northwest University. Xi'an, 710069, P. R. China.

\$Shaanxi Institute of International Trade\& Commerce, Xi'an, 712046, P. R. China.

*E-mail: 1hou2009@nwu.edu.cn 


\section{Section 1. Instruments and Methods}

Powder X-ray diffraction (PXRD) data were collected on a Bruker D8 ADVANCE X-ray powder diffractometer. Infrared (IR) spectra $\left(400-4000 \mathrm{~cm}^{-1}\right)$ were recorded through an EQUINOX-55 FT-IR spectrometer. Elemental analyses were performed with a Perkin-Elmer 2400C Elemental analyzer. Inductively coupled plasma (ICP) spectroscopy was performed on an Agilent 725 ICP-OES spectrometer. Thermogravimetric analyses (TGA) were carried out in $\mathrm{N}_{2}$ atmosphere using a Netzsch TG209F3 equipment at a heating rate of $10{ }^{\circ} \mathrm{C} \mathrm{min}{ }^{-1}$. Electrochemical behaviors were evaluated on an electrochemical workstation (CHI630E) with three-electrode system containing a modified GCE $(\varnothing 3 \mathrm{~mm})$ as the working electrode, a saturated calomel electrode as the reference electrode and a Pt wire as the counter electrode. X-ray photoelectron spectroscopy (XPS) measurement was performed on an AXIS Ultra spectrometer. Scanning electron microscopy (SEM) analysis was carried out on an SU8010 Field Emission Scanning Electron Microscope. Transmission electron microscope (TEM) analysis was collected using a JEOL JEM-2100 Plus Transmission Electron Microscope. Magnetic data were collected on a Quantum Design MPMS-XL-7 SQUID magnetometer.

\section{Section 2. X-Ray Crystallographic Measurements}

Single crystal diffraction data were collected on a Bruker SMART APEX II CCD detector at 153(2) K using Mo Ka radiation source. The structure was solved by direct methods and refined by using SHELXL-2014. ${ }^{1}$ The non-H atoms were refined anisotropically, while the $\mathrm{H}$ atoms were refined isotropically. The SQUEEZE was used in refinement to exclude the effect from the disordered solvent molecules. The crystallographic data were shown in Table S1, and selected bond lengths and angles were listed in Table S2.

(1) Sheldrick, G. M., SHELXL-2014, Program for Crystal Structure Refinement, University of Göttingen, Germany, 2014. 


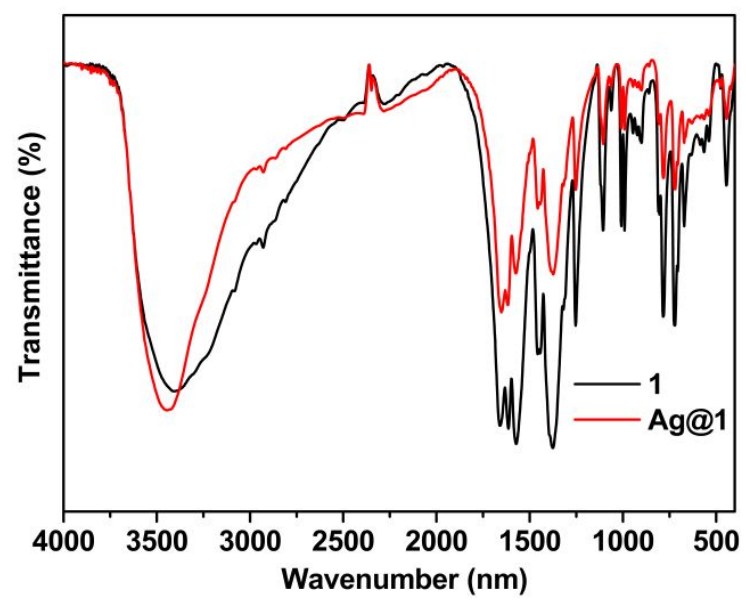

Figure S1. IR curves for 1 and Ag@1.

(a)

(b)

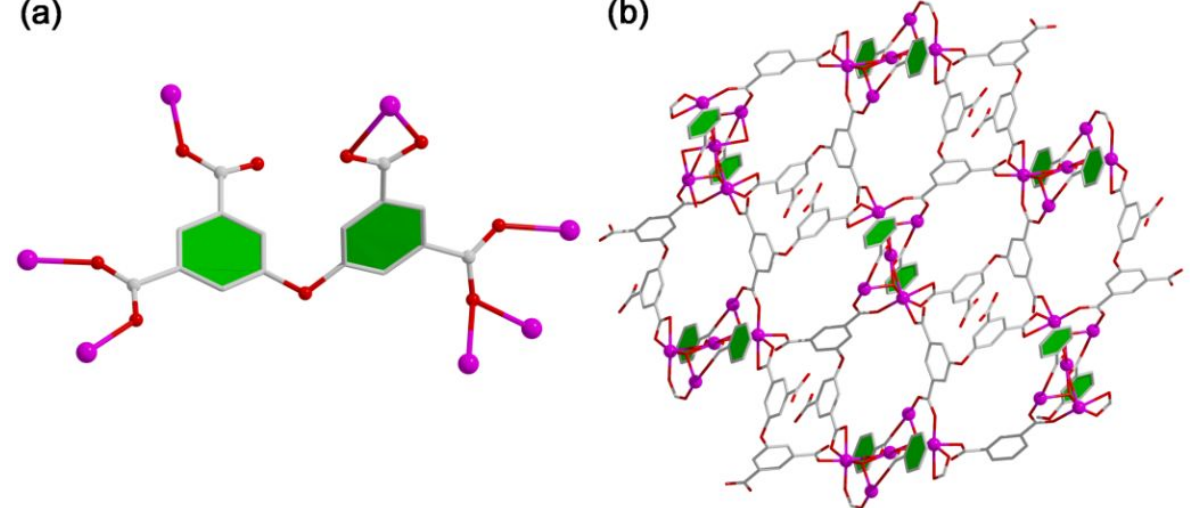

Figure S2. (a) Coordination modes of $\mathrm{L}^{4-}$ ligand in 1, and (b) 2D layer structure.

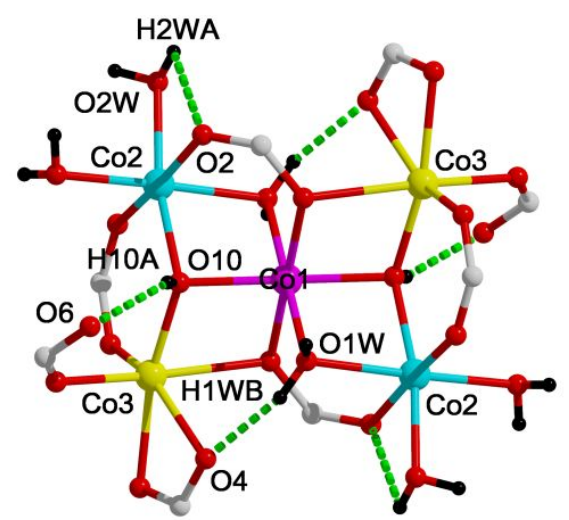

Figure S3. Intramolecular H-bonding interactions between the carboxylate groups and $\mathrm{H}_{2} \mathrm{O}$ molecules or $\mathrm{OH}^{-}$anions in $\mathbf{1}$. 


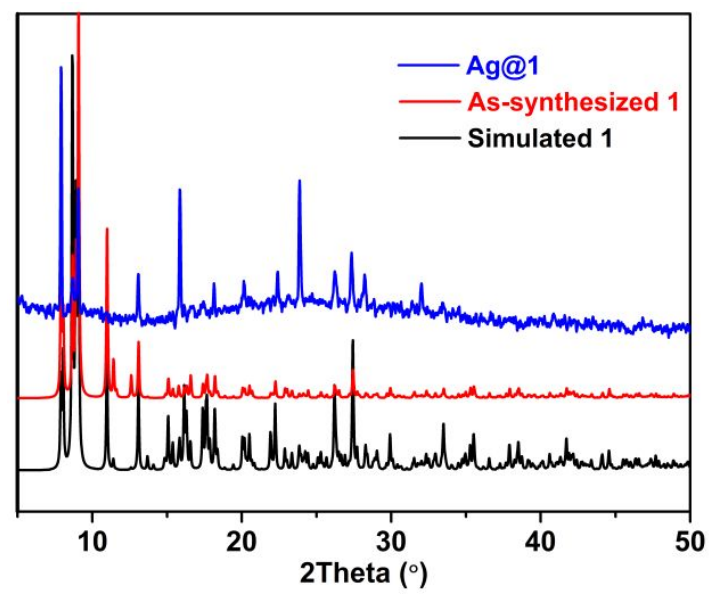

Figure S4. PXRD patterns of the simulated pattern from single-crystal structure, as-synthesized sample and synthesized Ag@1 composite.

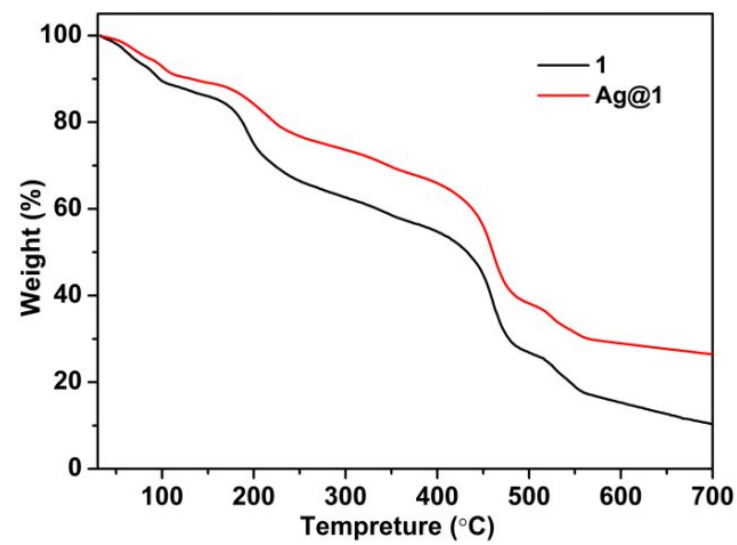

Figure S5. TGA curves for 1 and $\operatorname{Ag@1.~}$

(a)

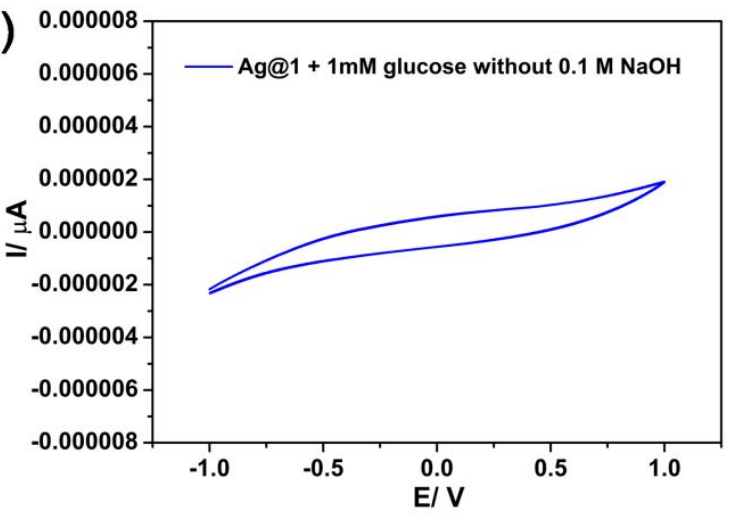

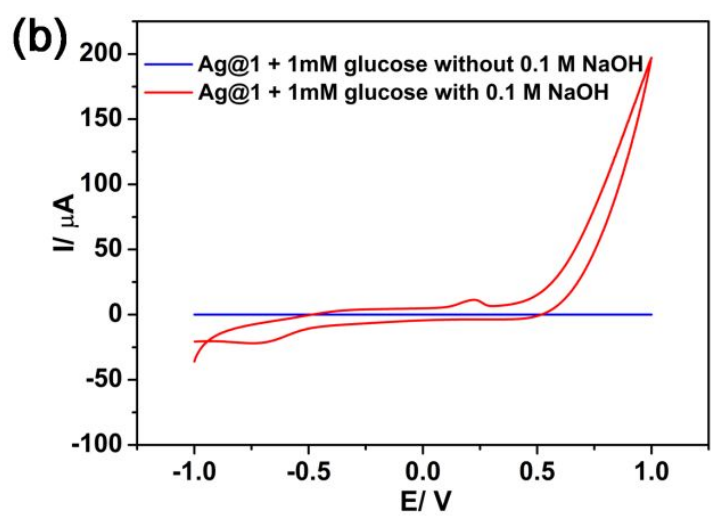

Figure S6. (a) CVs of $\mathrm{Ag} @ 1$ modified electrode without $0.1 \mathrm{M} \mathrm{NaOH}$ (scan rate $=50$ $\left.\mathrm{mVs}^{-1}\right)$. (b) comparison of $\mathrm{Ag} @ 1$ modified electrode with and without $0.1 \mathrm{M} \mathrm{NaOH}$ $\left(\right.$ scan rate $\left.=50 \mathrm{mVs}^{-1}\right)$. 


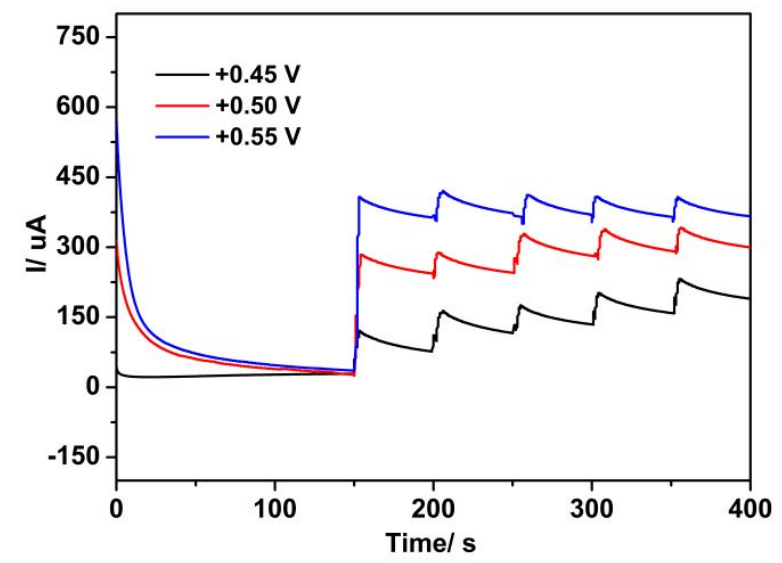

Figure S7. Chronoamperometric responses of $\mathrm{Ag} @ 1$ in $0.1 \mathrm{M} \mathrm{NaOH}$ upon successive addition of $0.1 \mathrm{mM}$ glucose.

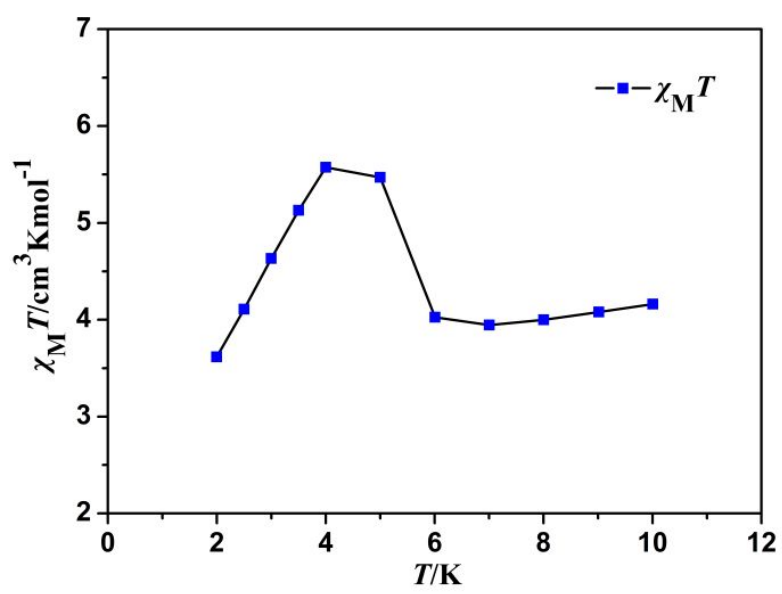

Figure S8. $\chi_{\mathrm{M}} T$ vs. $T$ plot for $\mathbf{1}$ in the temperature of $2-10 \mathrm{~K}$.

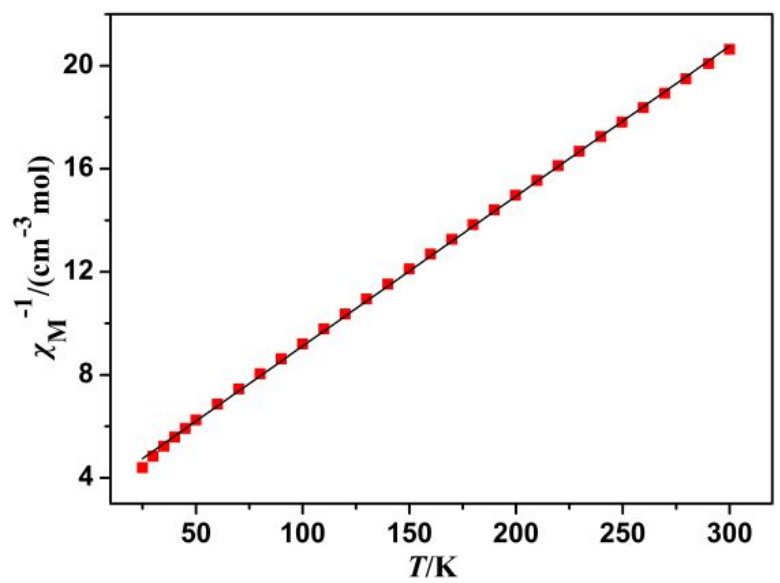

Figure S9. $\chi_{\mathrm{M}}{ }^{-1} v s . T$ plot fitted by the Curie-Weiss law for $\mathbf{1}$. 


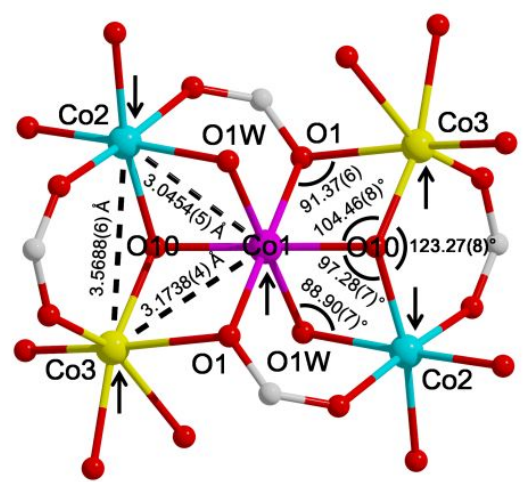

Figure S10. Bond lengths and bond angles in $\mathrm{Co}_{5}$ cluster unit.

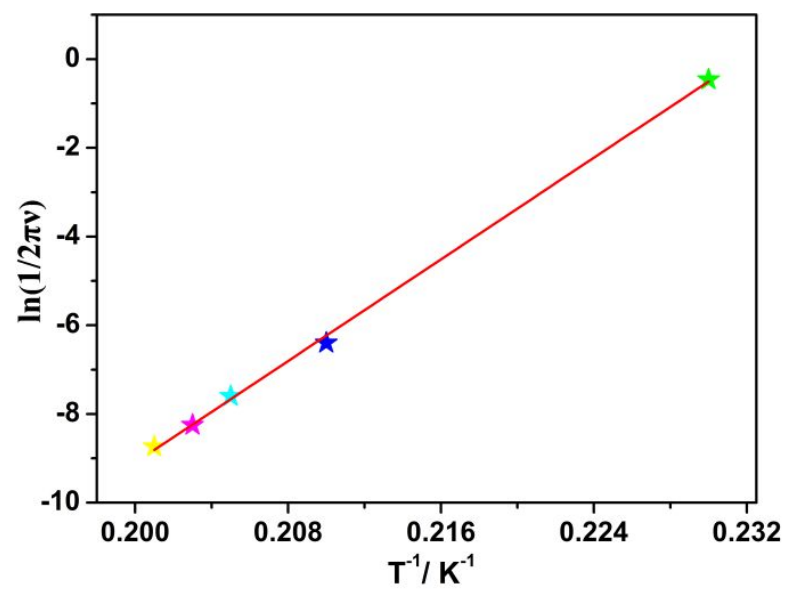

Figure S11. Arrhenius plot and best linear fit for $\mathbf{1}$ at zero dc field.

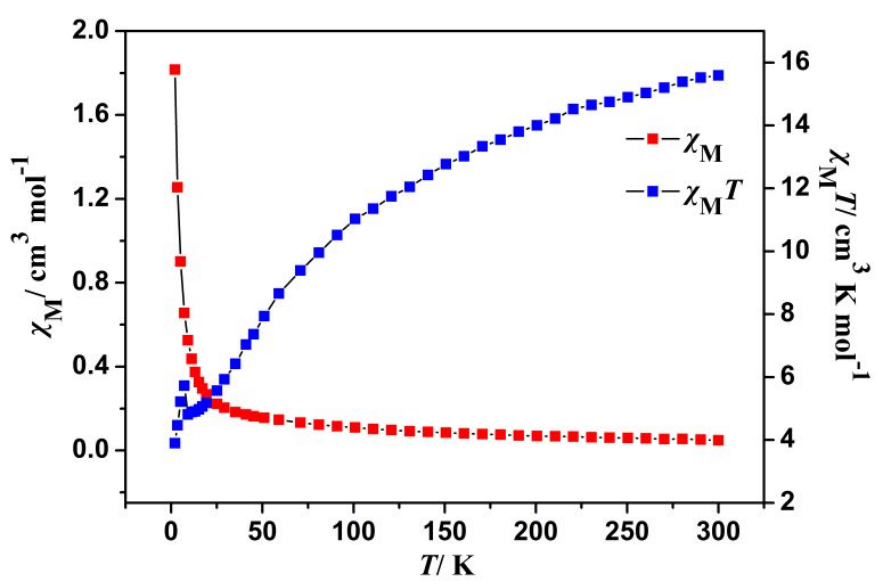

Figure S12. $\chi_{\mathrm{M}}$ and $\chi_{\mathrm{M}} T v s . T$ plots for $\mathrm{Ag} @ \mathbf{1}$ 


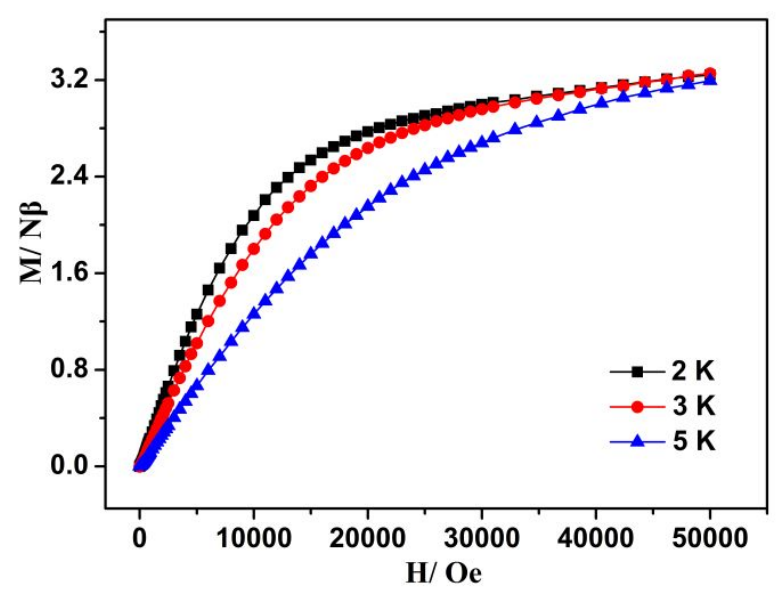

Figure S13. $M v s . H$ plots for Ag@1 at 2.0,3.0, and 5.0 K.

Table S1. Crystallographic data and structure refinement parameters for $\mathbf{1}$.

\begin{tabular}{ll}
\hline complex & $\mathbf{1}$ \\
chemical formula & $\mathrm{C}_{32} \mathrm{H}_{26} \mathrm{Co}_{5} \mathrm{O}_{26}$ \\
formula weight & 1121.18 \\
$T(\mathrm{~K})$ & $153(2)$ \\
crystal system & Monoclinic \\
space group & $C 2 / \mathrm{c}$ \\
$a(\AA)$ & $19.9632(18)$ \\
$b(\AA)$ & $13.5266(18)$ \\
$c(\AA)$ & $22.045(2)$ \\
$\alpha(\mathrm{deg})$ & 90 \\
$\beta(\mathrm{deg})$ & $95.648(5)$ \\
$\gamma(\mathrm{deg})$ & 90 \\
$V\left(\AA^{3}\right)$ & $5924.0(11)$ \\
$Z$ & 4 \\
$D_{\mathrm{c}}\left(\mathrm{mg} / \mathrm{m}^{3}\right)$ & 1.257 \\
$\left.\mu(\mathrm{mm})^{-1}\right)$ & 1.437 \\
reflns collected & 69839 \\
\hline
\end{tabular}




\begin{tabular}{|c|c|}
\hline GOF & 1.050 \\
\hline$R_{\text {int }}$ & 0.0758 \\
\hline$R_{1}^{\mathrm{a}}, w R_{2}^{\mathrm{b}}[I>2 \sigma]$ & $0.0324,0.0716$ \\
\hline$R_{1}, w R_{2}$ (all data) & $0.0423,0.0766$ \\
\hline
\end{tabular}

Table S2. Selected bond lengths $(\AA)$ and angles (deg) for $\mathbf{1}$.

\begin{tabular}{|c|c|c|c|}
\hline \multicolumn{4}{|c|}{1} \\
\hline $\mathrm{Co}(1)-\mathrm{O}(10)$ & $2.0084(16)$ & $\mathrm{Co}(2)-\mathrm{O}(2) \# 3$ & $2.0953(18)$ \\
\hline $\mathrm{Co}(1)-\mathrm{O}(10) \# 1$ & $2.0085(16)$ & $\mathrm{Co}(2)-\mathrm{O}(3 \mathrm{~W})$ & $2.110(2)$ \\
\hline $\mathrm{Co}(1)-\mathrm{O}(1) \# 2$ & $2.0836(16)$ & $\mathrm{Co}(2)-\mathrm{O}(1 \mathrm{~W})$ & $2.1957(18)$ \\
\hline $\mathrm{Co}(1)-\mathrm{O}(1) \# 3$ & $2.0836(17)$ & $\mathrm{Co}(3)-\mathrm{O}(9) \# 4$ & $2.0021(18)$ \\
\hline $\mathrm{Co}(1)-\mathrm{O}(1 \mathrm{~W})$ & $2.1530(17)$ & $\mathrm{Co}(3)-\mathrm{O}(10)$ & $2.0067(17)$ \\
\hline $\mathrm{Co}(1)-\mathrm{O}(1 \mathrm{~W}) \# 1$ & $2.1531(17)$ & $\mathrm{Co}(3)-\mathrm{O}(7) \# 5$ & $2.0418(18)$ \\
\hline $\mathrm{Co}(2)-\mathrm{O}(8) \# 4$ & $2.0356(18)$ & $\mathrm{Co}(3)-\mathrm{O}(3)$ & $2.1455(19)$ \\
\hline $\mathrm{Co}(2)-\mathrm{O}(10)$ & $2.0493(18)$ & $\mathrm{Co}(3)-\mathrm{O}(4)$ & $2.2453(19)$ \\
\hline $\mathrm{Co}(2)-\mathrm{O}(2 \mathrm{~W})$ & $2.091(2)$ & $\mathrm{Co}(3)-\mathrm{O}(1) \# 2$ & $2.3449(18)$ \\
\hline $\mathrm{O}(10)-\mathrm{Co}(1)-\mathrm{O}(10) \# 1$ & $180.00(14)$ & $\mathrm{O}(2 \mathrm{~W})-\mathrm{Co}(2)-\mathrm{O}(3 \mathrm{~W})$ & $94.59(10)$ \\
\hline $\mathrm{O}(10)-\mathrm{Co}(1)-\mathrm{O}(1) \# 2$ & $83.64(7)$ & $\mathrm{O}(2) \# 3-\mathrm{Co}(2)-\mathrm{O}(3 \mathrm{~W})$ & $88.82(8)$ \\
\hline $\mathrm{O}(10) \# 1-\mathrm{Co}(1)-\mathrm{O}(1) \# 2$ & $96.36(7)$ & $\mathrm{O}(8) \# 4-\mathrm{Co}(2)-\mathrm{O}(1 \mathrm{~W})$ & $88.96(8)$ \\
\hline $\mathrm{O}(10)-\mathrm{Co}(1)-\mathrm{O}(1) \# 3$ & $96.36(7)$ & $\mathrm{O}(10)-\mathrm{Co}(2)-\mathrm{O}(1 \mathrm{~W})$ & $82.76(7)$ \\
\hline $\mathrm{O}(10) \# 1-\mathrm{Co}(1)-\mathrm{O}(1) \# 3$ & $83.64(7)$ & $\mathrm{O}(2 \mathrm{~W})-\mathrm{Co}(2)-\mathrm{O}(1 \mathrm{~W})$ & $87.97(8)$ \\
\hline $\mathrm{O}(1) \# 2-\mathrm{Co}(1)-\mathrm{O}(1) \# 3$ & $180.00(3)$ & $\mathrm{O}(2) \# 3-\mathrm{Co}(2)-\mathrm{O}(1 \mathrm{~W})$ & $89.98(7)$ \\
\hline $\mathrm{O}(10)-\mathrm{Co}(1)-\mathrm{O}(1 \mathrm{~W})$ & $84.80(7)$ & $\mathrm{O}(3 \mathrm{~W})-\mathrm{Co}(2)-\mathrm{O}(1 \mathrm{~W})$ & 177.09(9) \\
\hline $\mathrm{O}(10) \# 1-\mathrm{Co}(1)-\mathrm{O}(1 \mathrm{~W})$ & $95.20(7)$ & $\mathrm{O}(9) \# 4-\mathrm{Co}(3)-\mathrm{O}(10)$ & $102.76(7)$ \\
\hline $\mathrm{O}(1) \# 2-\mathrm{Co}(1)-\mathrm{O}(1 \mathrm{~W})$ & $91.42(7)$ & $\mathrm{O}(9) \# 4-\mathrm{Co}(3)-\mathrm{O}(7) \# 5$ & $97.16(8)$ \\
\hline $\mathrm{O}(1) \# 3-\mathrm{Co}(1)-\mathrm{O}(1 \mathrm{~W})$ & $88.58(7)$ & $\mathrm{O}(10)-\mathrm{Co}(3)-\mathrm{O}(7) \# 5$ & $93.75(7)$ \\
\hline $\mathrm{O}(10)-\mathrm{Co}(1)-\mathrm{O}(1 \mathrm{~W}) \# 1$ & $95.20(7)$ & $\mathrm{O}(9) \# 4-\mathrm{Co}(3)-\mathrm{O}(3)$ & $93.35(7)$ \\
\hline $\mathrm{O}(10) \# 1-\mathrm{Co}(1)-\mathrm{O}(1 \mathrm{~W}) \# 1$ & $84.80(7)$ & $\mathrm{O}(10)-\mathrm{Co}(3)-\mathrm{O}(3)$ & $163.37(7)$ \\
\hline $\mathrm{O}(1) \# 2-\mathrm{Co}(1)-\mathrm{O}(1 \mathrm{~W}) \# 1$ & $88.58(7)$ & $\mathrm{O}(7) \# 5-\mathrm{Co}(3)-\mathrm{O}(3)$ & $88.25(8)$ \\
\hline
\end{tabular}




\begin{tabular}{llll}
\hline $\mathrm{O}(1) \# 3-\mathrm{Co}(1)-\mathrm{O}(1 \mathrm{~W}) \# 1$ & $91.42(7)$ & $\mathrm{O}(9) \# 4-\mathrm{Co}(3)-\mathrm{O}(4)$ & $150.41(7)$ \\
$\mathrm{O}(1 \mathrm{~W})-\mathrm{Co}(1)-\mathrm{O}(1 \mathrm{~W}) \# 1$ & 180 & $\mathrm{O}(10)-\mathrm{Co}(3)-\mathrm{O}(4)$ & $103.64(7)$ \\
$\mathrm{O}(8) \# 4-\mathrm{Co}(2)-\mathrm{O}(10)$ & $95.35(7)$ & $\mathrm{O}(7) \# 5-\mathrm{Co}(3)-\mathrm{O}(4)$ & $94.24(8)$ \\
$\mathrm{O}(8) \# 4-\mathrm{Co}(2)-\mathrm{O}(2 \mathrm{~W})$ & $88.96(8)$ & $\mathrm{O}(3)-\mathrm{Co}(3)-\mathrm{O}(4)$ & $59.74(7)$ \\
$\mathrm{O}(10)-\mathrm{Co}(2)-\mathrm{O}(2 \mathrm{~W})$ & $169.70(8)$ & $\mathrm{O}(9) \# 4-\mathrm{Co}(3)-\mathrm{O}(1) \# 2$ & $93.36(7)$ \\
$\mathrm{O}(8) \# 4-\mathrm{Co}(2)-\mathrm{O}(2) \# 3$ & $174.90(8)$ & $\mathrm{O}(10)-\mathrm{Co}(3)-\mathrm{O}(1) \# 2$ & $77.25(6)$ \\
$\mathrm{O}(10)-\mathrm{Co}(2)-\mathrm{O}(2) \# 3$ & $89.47(7)$ & $\mathrm{O}(7) \# 5-\mathrm{Co}(3)-\mathrm{O}(1) \# 2$ & $167.47(7)$ \\
$\mathrm{O}(2 \mathrm{~W})-\mathrm{Co}(2)-\mathrm{O}(2) \# 3$ & $86.02(8)$ & $\mathrm{O}(3)-\mathrm{Co}(3)-\mathrm{O}(1) \# 2$ & $97.93(7)$ \\
$\mathrm{O}(8) \# 4-\mathrm{Co}(2)-\mathrm{O}(3 \mathrm{~W})$ & $92.46(9)$ & $\mathrm{O}(4)-\mathrm{Co}(3)-\mathrm{O}(1) \# 2$ & $79.70(7)$ \\
$\mathrm{O}(10)-\mathrm{Co}(2)-\mathrm{O}(3 \mathrm{~W})$ & $94.58(9)$ & $\mathrm{Co}(1)-\mathrm{O}(10)-\mathrm{Co}(2)$ & $97.28(7)$ \\
$\mathrm{Co}(3)-\mathrm{O}(10)-\mathrm{Co}(2)$ & $123.27(8)$ & $\mathrm{Co}(3)-\mathrm{O}(10)-\mathrm{Co}(1)$ & $104.46(8)$ \\
$\mathrm{Co}(1)-\mathrm{O}(1 \mathrm{~W})-\mathrm{Co}(2)$ & $88.90(7)$ & $\mathrm{Co}(1)-\mathrm{O}(1)-\mathrm{Co}(3)$ & $91.37(6)$
\end{tabular}

Symmetry codes: For 1, \#1 -x+1/2, -y+3/2, $-\mathrm{z}+1$; \#2 -x, $-\mathrm{y}+1,-\mathrm{z}+1$; \#3 x+1/2, y+1/2, $\mathrm{z}$; $\# 4 \mathrm{x},-\mathrm{y}+1, \mathrm{z}+1 / 2 ; \# 5-\mathrm{x}+1 / 2,-\mathrm{y}+1 / 2,-\mathrm{z}+1$.

Table S3. Hydrogen bond lengths $(\AA)$ and bond angles (deg) for $\mathbf{1}$.

\begin{tabular}{lllll}
\hline $\mathrm{D}-\mathrm{H} \cdots \mathrm{A}$ & $\mathrm{D}-\mathrm{H}(\AA)$ & $\mathrm{H} \cdots \mathrm{A}(\AA)$ & $\mathrm{D} \cdots \mathrm{A}(\AA)$ & $\mathrm{D}-\mathrm{H} \cdots \mathrm{A}(\mathrm{deg})$ \\
\hline $\mathrm{O} 1 \mathrm{~W}-\mathrm{H} 1 \mathrm{WB} \cdots \mathrm{O} 4$ & 0.85 & 1.97 & $2.7744(4)$ & 156 \\
$\mathrm{O} 2 \mathrm{~W}-\mathrm{H} 2 \mathrm{WA} \cdots \mathrm{O} 2$ & 0.85 & 2.57 & $2.8556(4)$ & 101 \\
$\mathrm{O} 10-\mathrm{H} 10 \mathrm{~A} \cdots \mathrm{O} 6$ & 0.82 & 2.07 & $2.7393(4)$ & 139 \\
\hline
\end{tabular}

Table S4. ICP analyses for 1 and Ag@1.

\begin{tabular}{lll}
\hline Samples & Amount of $\mathrm{Co}^{2+}(\%)$ & ${\text { Amount of } \mathrm{Ag}^{+}(\%)}^{(}{ }^{2}$ \\
\hline $\mathbf{1}$ & 22.9 & 0 \\
$\mathrm{Ag} @ \mathbf{1}$ & 22.5 & 1.8 \\
\hline
\end{tabular}

